\title{
Shear enhanced heterogeneous nucleation in some Mg- and Al-alloys
}

\author{
Z. Fan ${ }^{1}$, Y. Wang ${ }^{1}$, Z. F. Zhang ${ }^{1,2}$, M. Xia ${ }^{1}$, H. T. Li ${ }^{1}$, J. Xu' ${ }^{2}$, L. Granasy ${ }^{1}$ and \\ G. M. Scamans ${ }^{1}$
}

Intensive shearing was applied to alloy melts at temperatures above their liquidus by using a twinscrew mechanism. The sheared melt was then cast into a TP1 mould for microstructural examination. Alloy melts with or without shearing were also filtered using the Prefil technique developed by N-Tech Ltd in order to analyse oxides and other second phase particles. The experimental results showed a significant grain refinement through enhancement of heterogeneous nucleation. The intensive melt shearing converted oxide films and agglomerates into well dispersed fine particles with a narrow size distribution. It was confirmed that the fine oxide particles can act as potent sites for nucleation during the solidification of the sheared melt. This paper presents the experimental results and theoretical analysis of shear enhanced heterogeneous nucleation during solidification of $\mathrm{Mg}$ - and Al-alloys. A multi-step heterogeneous nucleation mechanism has been proposed and discussed.

Keywords: Solidification, Heterogeneous Nucleation, Grain Refinement

\section{Introduction}

It is well accepted that a fine grain size usually results in an increase in mechanical properties, such as ambient temperature strength, toughness and ductility. An effective method to achieve a fine grain structure is by controlled solidification using grain refiner to promote heterogeneous nucleation. A typical example is the inoculation in $\mathrm{Al}$ alloys, with particles based on the Al-Ti-B and Al-Ti-C systems. The phases $\mathrm{TiB}_{2}$ and TiC are believed to be potent substrates on which $\mathrm{Al}$ grains nucleate heterogeneously. ${ }^{1-3}$ In spite of the large volume of work carried out on grain refinement over the past half century, significant challenges remain, ${ }^{3}$ such as (i) the very low refinement efficiency, i.e., only $0 \cdot 1-1 \%$ of the added particles are effective; (ii) agglomeration of the refiner particles, (iii) restricted applicability due to the so-call poisoning effects in the presence of certain solute elements, and (iv) the detrimental effect of nonnucleating refiner particles on the subsequent thermal mechanical alloy processing. In recent decades, tremendous progress has been made in the understanding of growth kinetics in solidification, which has led to advances in the predictive modelling of the as-solidified microstructure. Unfortunately this modelling was mainly based on the growth competition with little or no attention to the nucleation process. The mathematical description of growth kinetics has not been matched by similar progress for nucleation

${ }^{1}$ BCAST, Brunel University, Uxbridge, Middlesex UB8 3PH, UK

${ }^{2}$ General Research Institute for Nonferrous Metals, Beijing, 100088, China

e-mail: yun.wang@brunel.ac.uk kinetics ${ }^{4}$ due to the difficulties of making nucleation investigations.

We have found that an improvement in microstructural and compositional uniformity with significant grain refinement for both $\mathrm{Mg}$ - and Al-alloys by using an MCAST (melt conditioning by advanced shear technology) process developed recently. ${ }^{4-7}$ The intensive shearing imposed to the liquid metal significantly changes nucleation behaviour and the cast microstructure. In the present paper, we present the experimental results and theoretical analysis of shear enhanced heterogeneous nucleation during solidification of $\mathrm{Mg}$ and Al-alloys. The experimental results from this work will be discussed in terms of the heterogeneous nucleation mechanism of the sheared melt, in comparison to conventional grain refiners.

\section{Experimental}

AD91D, A380 and Al-10Zn-2Mg alloys (see Table 1 for actual compositions) were melted at $675^{\circ} \mathrm{C}, 700^{\circ} \mathrm{C}$ and $750^{\circ} \mathrm{C}$, respectively. The melt was then transferred to an MCAST unit and subjected to 45 seconds of intensive shearing using a pair of screws with a fixed rotation speed of $800 \mathrm{rpm}$. The casting temperature ranged from $605^{\circ} \mathrm{C}$ to $650^{\circ} \mathrm{C}$ for AZ91D. The detailed description of the shearing mechanism of the MCAST unit can be found elsewhere. ${ }^{4-7}$ The Aluminium Association TP1 mould $^{8}$ was used for casting the sheared and nonsheared melts under similar conditions. A pressurised filtration technique $\left(\right.$ Prefil $^{\circledR}, \mathrm{N}-\mathrm{Tec}$ Ltd, UK) was used to collect oxides and other inclusions for optical and scanning electron microscopy.

(c) 2009 W. S. Maney \& Son Ltd.

Received 17 June 2008; accepted 12 September 2008 DOI

International Journal of Cast Metals Research 2009 VOL 22 NO 1-4

International Journal of Cast Metals Research ijcFanWang.3d 9/10/08 17:22:35

The Charlesworth Group, Wakefield +44(0)1924 369598 - Rev $7.51 \mathrm{n} / \mathrm{W}$ (Jan 202003$)$ 
The TP1 ingots were cut at a height of $38 \mathrm{~mm}$ from the base, corresponding to a cooling rate of $3.5 \mathrm{~K} / \mathrm{s}$. Following the Prefil experiment, the residual alloy which remained above the filter was sectioned together with the filter. The specimens for optical microscopy (OM) and scanning electron microscopy (SEM) were prepared using standard polishing procedures. A Zeiss imaging system was used for the $\mathrm{OM}$ and the quantitative measurements of the microstructural features. A colour etching technique for $\mathrm{Mg}$ alloy and an anodising technique for Al alloys were also used. SEM was carried out using an FEG Zeiss Supera microscope, equipped with an energy dispersive spectroscopy (EDS) X-ray microanalysis facility.

\section{Results}

\section{Effect of shearing on grain size}

Figure 1 shows the microstructure of the AZ91D Mgalloy, with and without melt shearing, cast at $650^{\circ} \mathrm{C}$ and $605^{\circ} \mathrm{C}$ in the TP1 mould showing the significant grain refinement provided by the intensive shearing. The mean grain size decreased from $690 \mu \mathrm{m}$ to $175 \mu \mathrm{m}$ at $650^{\circ} \mathrm{C}$. It was found that this grain refinement was effective over the whole casting temperature range studied for the AZ91D, i.e., $605^{\circ} \mathrm{C} \sim 650^{\circ} \mathrm{C}$, Figure 2. The influence of the melt superheat on the grain size was suppressed by the shearing effect, with the mean grain size varying from $85 \mu \mathrm{m}$ to $175 \mu \mathrm{m}$ over the temperature range when intensive shearing was imposed, compared to $210 \mu \mathrm{m}$ to $690 \mu \mathrm{m}$ without melt shearing. The equivalent grain refinement by the intensive shearing of the Al-10.5Zn$2 \mathrm{Mg}$ alloy is shown in Figure 3. In this case, the mean grain size decreased from $247 \mu \mathrm{m}$ to $44 \mu \mathrm{m}$ when the sheared Al-alloy melt was cast in the TP1 mould at $650^{\circ} \mathrm{C}$.

\section{Oxides and intermetallic particles in alloy melt}

The uniform, fine and equiaxed microstructure is promoted by a high rate of heterogeneous nucleation in the sheared melt. This requires numerous potent inoculants to have been present before solidification begins. SEM of the Al-10Zn-2Mg alloy shows the presence of $\mathrm{Al}_{3} \mathrm{Ti}$ as the inoculant particles, Figure 4a. Detailed SEM of large number of the $\mathrm{Al}_{3}$ Ti particles showed oxide particles inside most if not all particles, indicating that the intermetallic particles were apparently nucleated on the oxides. To clarify this phenomenon, AZ91D Mg alloy and LM24 Al-alloy (Al-9Si$3 \mathrm{Cu}-0 \cdot 5 \mathrm{Mg}$ ) were also examined. Figures 3(b) shows the morphology of $\mathrm{Al}_{8} \mathrm{Mn}_{5}$ particles in the AZ91D alloy. Blocky $\alpha$-AlFeMnSi phase was also found in LM24 alloy. $\mathrm{Al}_{8} \mathrm{Mn}_{5}$ particles are seen to be similar in size with the mean diameter being $4.3 \mu \mathrm{m}$, compared to $7.9 \mu \mathrm{m}$ for $\alpha$-AlFeMnSi in the LM24 alloy. Oxide particles were frequently observed inside these compounds, showing that they probably nucleated on the oxides particles.

\section{Discussion}

The present work has demonstrated that melt-shearing exerts a significant influence on solidification behaviour during the casting process, resulting in a fine uniform microstructure for both $\mathrm{Mg}$ - and Al-alloys. This grain refinement is related to the enhancement of heterogeneous nucleation by melt shearing. Usually liquid alloy melts contain oxide particle clusters and oxide films. The most common morphology for the oxide present in as-cast Al-alloys is double oxide films known as bifilms. ${ }^{9-10}$ It has been reported that the bifilms were favourable sites for the nucleation of a wide variety of intermetallics in many matrices, but not for the matrix phase itself. ${ }^{10}$ For instance, both $\alpha$-AlFeSi and $\beta$-AlFeSi phases probably precipitated on the wetted outside surfaces of the bifilms. ${ }^{11}$ Although the bifilms are able to act as the substrates on which some intermetallic phases nucleate heterogenerously, the efficiency of the nucleation enhancement is limited due to their morphology and non-dispersed distribution. With the intensive shearing imposed on the melt, the oxide films will be uniformly dispersive. More significantly, some of the oxide films can be broken-up into individual particles to provide morphology for more effective heterogeneous nucleation of the intermetallic phases.

Wettability is one of the essential conditions for a particle to act as a potent nucleating site. It has been reported that, in Al-alloys, primary $\alpha$-Al phase was not able to nucleate directly on oxides particles due to the non-wettability. ${ }^{9-10}$ To overcome this wetting problem, either forced wetting has to be promoted or an intermediate phase has to be found which wets the oxide particles. Apart from the reports of $\alpha$-AlFeSi and $\beta$-AlFeSi phases nucleating on bifilms, $\mathrm{Al}_{8} \mathrm{Mn}_{5}$ was found to play a key role in grain refinement in Alcontaining Mg-alloys. For instance, addition of $\mathrm{Mn}$ to AZ91D led a dramatic grain refinement due to the formation of $\mathrm{Al}_{8} \mathrm{Mn}_{5}$ particles, which acted as the potent particles for the nucleation of $\alpha-\mathrm{Mg} .{ }^{12}$ As shown in Figure 4, a large number of $\mathrm{Al}_{8} \mathrm{Mn}_{5}$ particles in the AZ91D Mg-alloy have been observed and found to nucleate on the oxide particles, as do the $\mathrm{Al}_{3} \mathrm{Ti}$ and $\alpha$ $\mathrm{AlFeMnSi}$ in Al-alloys. Studies have discussed the key role $\mathrm{Al}_{3}$ Ti plays in inoculation by $\mathrm{TiB}_{2}{ }^{13}$

Based on the previous studies and the experimental results in this work, a multi-step heterogeneous nucleation mechanism is proposed, as shown schematically in Figure 5. Intensive shearing of liquid metal disperses the oxide films and agglomerations into fine oxide particles with a narrow size distribution. Furthermore, intensive melt shearing can force the liquid metal to wet the dispersed oxide particles by overcoming the energy barrier for wetting. During the continuous cooling of the sheared melt, intermetallic phase (e.g., $\mathrm{Al}_{8} \mathrm{Mn}_{5}$ ) nucleates on the oxide particles. With further cooling to temperatures bellow the alloy liquidus, the primary phase (e.g., $\alpha-\mathrm{Mg}$ ) will then nucleate on the intermetallic

Table 1 Chemical composition (wt\%), liquidus $\left(T_{L}\right)$ and shearing temperature $\left(T_{S h}\right)$ for alloys tested in this work

\begin{tabular}{|c|c|c|c|c|c|c|c|c|c|c|}
\hline Alloy & $\mathrm{Si}$ & $\mathrm{Fe}$ & $\mathrm{Cu}$ & $\mathrm{Mn}$ & $\mathrm{Zn}$ & $\mathrm{Ti}$ & $\mathrm{Mg}$ & Al & $\mathrm{T}_{\mathrm{L}}\left({ }^{\circ} \mathrm{C}\right)$ & $\mathrm{T}_{\mathrm{Sh}}\left({ }^{\circ} \mathrm{C}\right)$ \\
\hline AZ91D & 0.03 & 0.002 & $<0.001$ & 0.22 & 0.67 & - & Bal. & $8 \cdot 8$ & 597 & $605-650$ \\
\hline LM24 & $9 \cdot 38$ & 0.80 & $2 \cdot 31$ & 0.26 & 1.02 & - & $0 \cdot 49$ & Bal. & 593 & 640 \\
\hline Al-10Zn-2Mg & $0 \cdot 15$ & 0.35 & $1 \cdot 6$ & - & $10 \cdot 5$ & 0.2 & $2 \cdot 0$ & Bal. & 628 & 650 \\
\hline
\end{tabular}




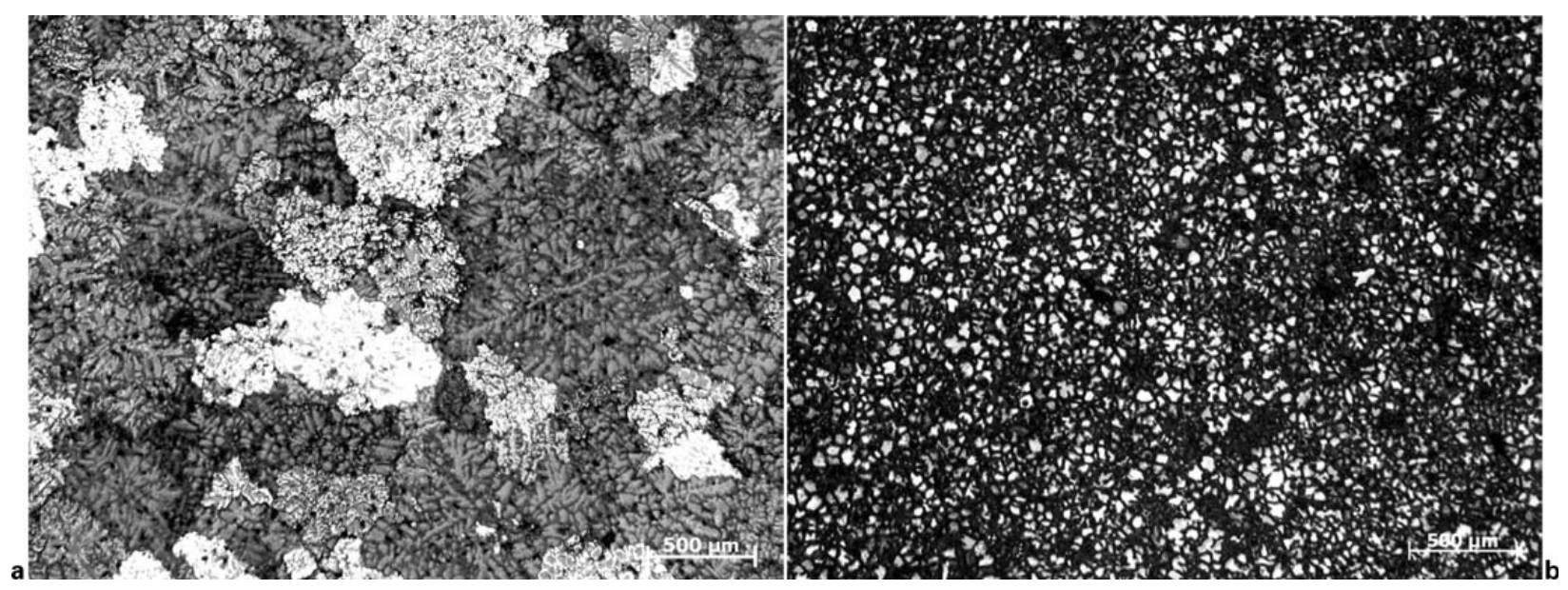

1 Microstructures of AZ91D alloy (a) without and (b) with intensive melt shearing cast in the TP1 mould (with $3.5 \mathrm{~K} / \mathrm{sec}$ cooling rate) at $650^{\circ} \mathrm{C}$ and $605^{\circ} \mathrm{C}$ respectively, showing the significant grain refinement with intensive melt shearing

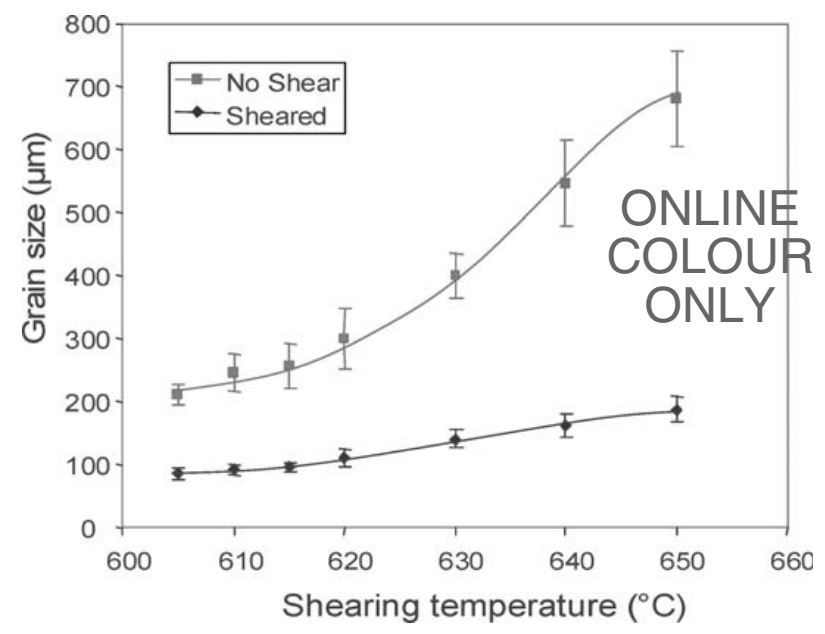

2 Variation of the grain size as a function of casting temperature for AZ91D Mg-alloy cast in TP1 mould with and without melt shearing
ONLINE COLOUR ONLY

particles. There is evidence that primary phase may also nucleate directly on the oxide particles if there is no intermetallic phases present.

The size distribution is critical for any particle to be active for heterogeneous nucleation. According to the free growth theory developed by Greer et al, ${ }^{14}$ the undercooling $\Delta T_{\mathrm{fg}}$ required for achieving the state of free growth is given by:

$$
\Delta T_{\mathrm{fg}}=4 \sigma_{\mathrm{lc}} /\left(\Delta S_{\mathrm{v}} d\right)
$$

where $d$ is diameter of the potent inoculant particles and $\Delta S_{\mathrm{v}}$ is the volumetric entropy of fusion. The free growth undercooling is inversely proportional to particle size, showing that the larger particles are more potent for heterogeneous nucleation and become active first and, under typical solidification conditions, only a small fraction of the particles become active before recalescence occurs. According to this theoretical model, more particles would contribute to heterogeneous nucleation if a narrow size distribution

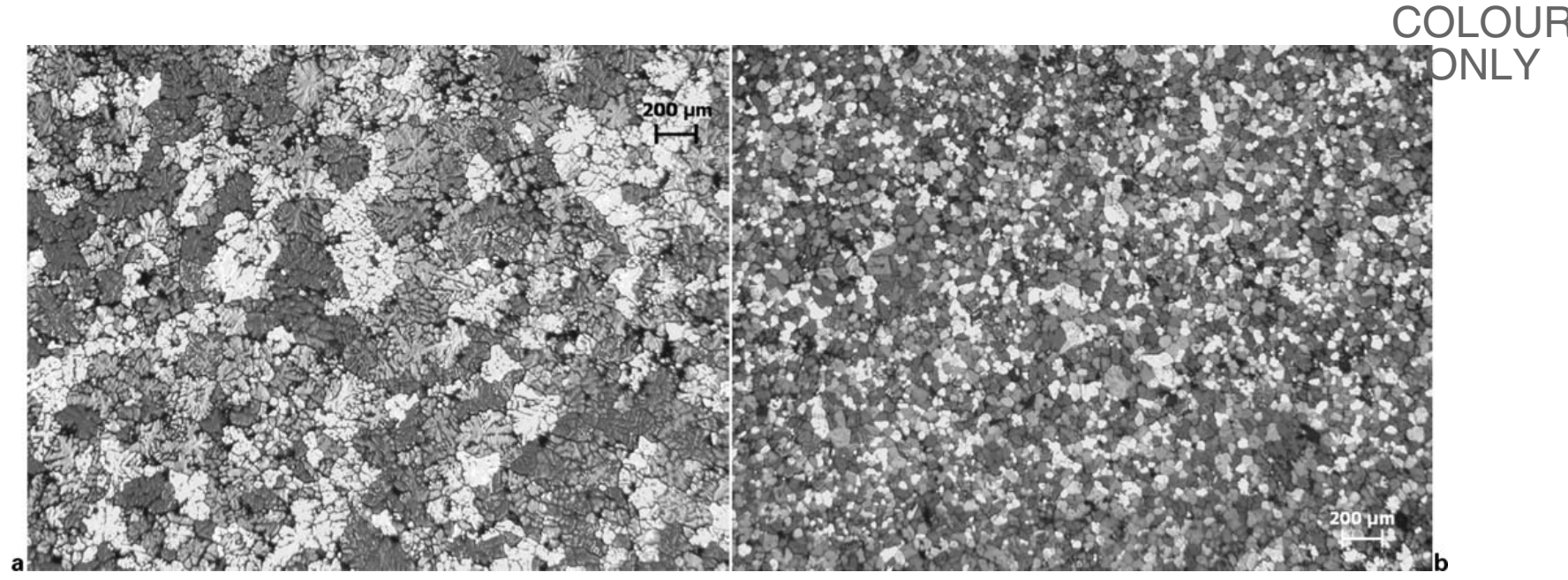

3 Microstructures of $\mathrm{Al}-10.5 \mathrm{Zn}-2 \mathrm{Mg}-1.6 \mathrm{Cu}-0.35 \mathrm{Fe}-0.15 \mathrm{Si}-0.2 \mathrm{Ti}$ Al-alloy cast at $650^{\circ} \mathrm{C}$ in the $\mathrm{TP} 1$ mould (a) without and (b) with intensive melt shearing, showing the significant grain refinement with intensive melt shearing 

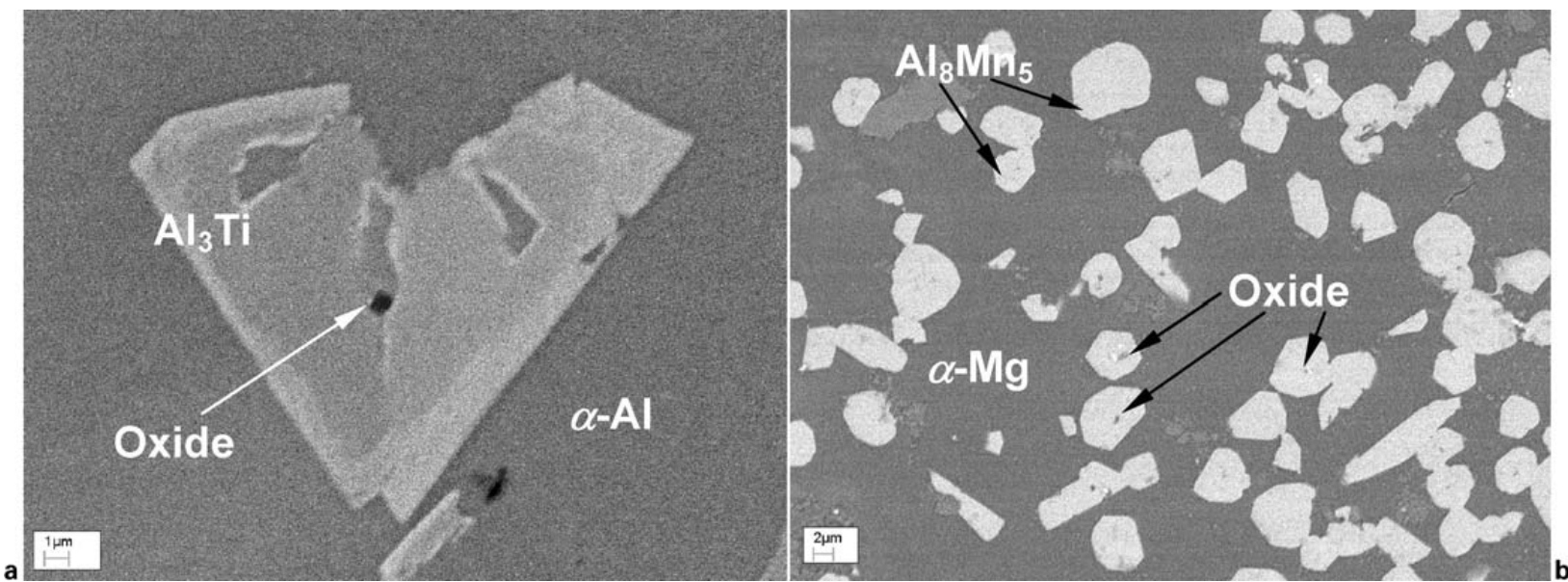

$4 \mathrm{SEM}$ micrographs showing the existence of the oxide particles inside (a) $\mathrm{Al}_{3} \mathrm{Ti}$ and (b) $\mathrm{Al}_{8} \mathrm{Mn}_{5}$ intermetallic particles in $\mathrm{Al}-10 \cdot 5 \mathrm{Zn}-2 \mathrm{Mg}-1 \cdot 6 \mathrm{Cu}-0.35 \mathrm{Fe}-0.15 \mathrm{Si}-0.2 \mathrm{Ti}$ and $\mathrm{AZ91D}$ alloys with intensive melt shearing, respectively. The particles in AZ91D alloy have been collected by a filtering technique

is achieved, and therefore the efficiency for the inoculation is promoted. In the present work, the intensive liquid metal shearing has been found to be effective for achieving fine oxide particles with a narrower size distribution. The intermetallics will be refined if they precipitate on the fine oxide particles, as observed previously for $\alpha-\mathrm{AlFeMnSi}{ }^{15}$ In contrast to the previous studies, oxides do promote the heterogeneous nucleation through the multi-step nucleation mechanism when the oxide particles are dispersed and of narrow size range. Although oxides are always present in alloy melts, this type of dramatic grain refinement had never been observed previously, due to non-wettability and the limited fraction of the activated particles. In the second step of the proposed nucleation mechanism, the intermetallic particles are similarly required to be of a narrow size spread in order to maximise the nucleation rate. These particles were very similar in size in all the alloys examined in the present work.

\section{Conclusions}

Intensive shearing, which is imposed directly to alloy melts at temperatures above the liquidus through a twinscrew mechanism, results in a significant grain refinement for $\mathrm{Mg}$ - and Al-alloys through enhancement of heterogeneous nucleation. The oxide films and agglomerations in melts are broken up and converted into fine well-dispersed particles with a very narrow size distribution. The fine oxide particles are potent sites for heterogeneous nucleation via the proposed multi-step nucleation mechanism, and the narrow spread in particle size, achieved by the melt-shearing, enables more particles to be active in the heterogeneous nucleation process.

\section{Acknowledgements}

The authors wish to thank Professors J. D. Hunt and P. V. Evans for their helpful discussions, MEL

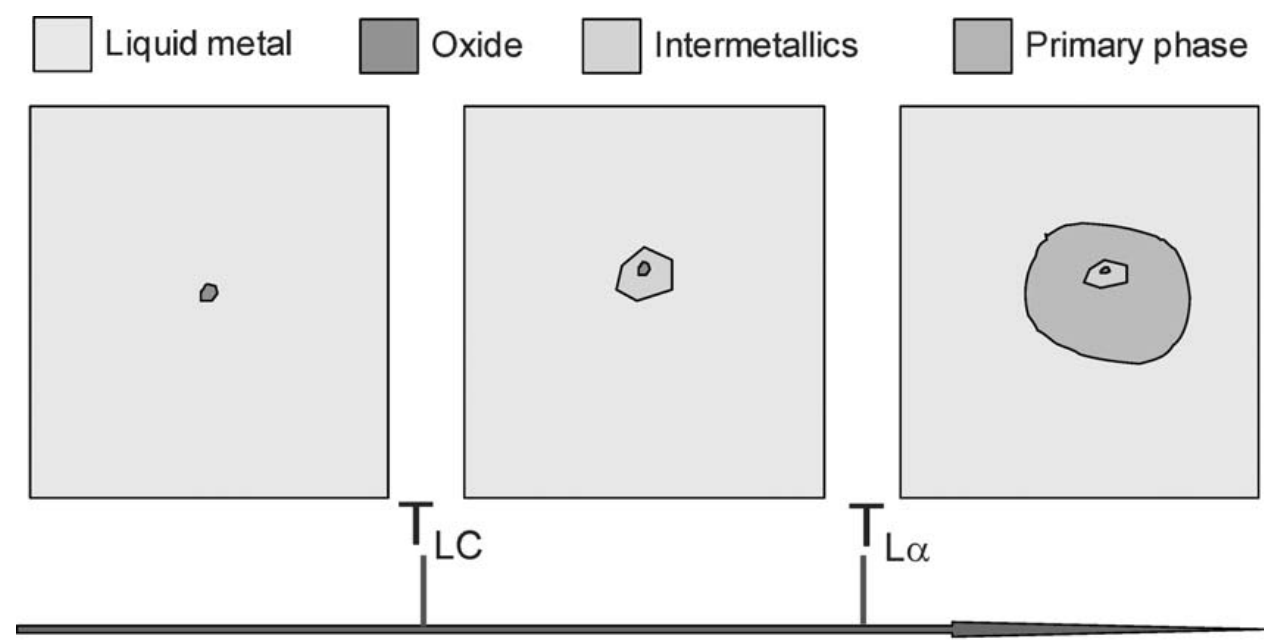

Decreasing Temperature

5 Schematic diagram showing the multi-step nucleation mechanism. $T_{L c}$ and $T_{L \alpha}$ denote the liquidus of intermetallic phase and the primary solid solution phase, respectively 
(Manchester, UK) for providing the AZ91D alloy ingots and Alcan (Voreppe, France) for providing the Al$10 \cdot 5 \mathrm{Zn}-2 \mathrm{Mg}-1 \cdot 6 \mathrm{Cu}-0 \cdot 35 \mathrm{Fe}-0 \cdot 15 \mathrm{Si}-0 \cdot 2 \mathrm{Ti}$ alloy ingots. Financial support from both EPSRC (UK) and TSB (UK) is gratefully acknowledged.

\section{References}

1. B. S. Murty, S. A. Kori and M. Chakraborty: Inter. Mater. Review, 2002, 47, 3 .

2. T. E. Quested: Mater. Sci. Tech., 2004, 20, 1357.

3. A. L. Greer: in 'Solidification and Casting', (ed. B. Cantor and K O'Reilly), Institute of Physics Publishing, 2003, p199.

4. Z. Fan and G. Liu: Acta Mater. 2005, 53, 4345.
5. Z. Fan, G. Liu and Y. Wang: J. Mater. Sci. 2006, 41, 3631.

6. Y. Wang, G. Liu and Z. Fan: Acta Mater. 2006, 54, 689.

7. M. Hitchcock, Y. Wang, Z. Fan: Acta Mater. 2007, 55, 1598.

8. Standard Test Procedures for Aluminium Alloy Grain Refiner: TP-

1. The Aluminium Association, Washington, DC, 1987.

9. J. Campbell: 'Castings', 2nd edn, Butterworth Heinemann 2003

10. J. Campbell: Mater. Sci. Tech., 2006, 22, 127.

11. X. Cao and J. Campbell: Metall. Mater. Trans. A, 2003, 34A, 1409.

12. Y. M. Kim, C. D. Yim, Y. H. Kim and B. S. You: in 'Magnesium Technology 2007', (ed. R. S. Beals et al.), TMS 2007, 121.

13. P Schumacher and A. L. Greer: Mater. Sci. Technol., 1998, 14, 394.

14. A. L. Greer, A. M. Bunn, A. Tronche, P. V. Evans and D. J. Bristow: Acta Mater., 2000, 48, 2823

15. X. Fang, G. Shao, Y. Q. Liu and Z. Fan: Mater. Sci. Eng. A, 2007, 445-446, 65 . 


\section{Authors Queries}

Journal: International Journal of Cast Metals Research

Paper: FanWang

Title: Shear enhanced heterogeneous nucleation in some Mg- and Al-alloys

Dear Author

During the preparation of your manuscript for publication, the questions listed below have arisen. Please attend to these matters and return this form with your proof. Many thanks for your assistance

\begin{tabular}{|l|l|l|}
\hline $\begin{array}{l}\text { Query } \\
\text { Reference }\end{array}$ & Query & Remarks \\
\hline 1 & Please confirm the short title. & \\
\hline
\end{tabular}

